\title{
The use of STEM imaging to analyze thickness variations due to electromigration-induced mass transport in thin polycrystalline nanobridges.
}

Maria Rudneva, Tatiana Kozlova and Henny W. Zandbergen

Kavli Institute of Nanoscience, Delft University of Technology, Lorentzweg 1, 2628 CJ, Delft, The Netherlands

Corresponding author: Maria Rudneva

Corresponding address: Lorentzweg 1, 2628 CJ, Delft, the Netherlands

\section{Highlights}

- We analyzed the electromigration-induced thickness variations of thin polycrystalline films.

- Small camera length is required to minimize the effect of diffraction contact in STEM imaging

- We correlated STEM signal with height measurements done with AFM

- We demonstrated applicability of STEM imaging for 3D characterization of nanoelectrodes.

\section{Keywords}

STEM, electromigration, polycrystalline nanobridges, mass transport, nanoelectrodes

\section{Abbreviations}

STEM - scanning transmission electron microscopy

AFM - atomic force microscopy

\section{Abstract}


Scanning transmission electron microscopy (STEM) imaging is applied to analyze the electromigration-induced thickness variations of thin polycrystalline films. It is shown that for a small camera length high-angle STEM (HASTEM) is required to minimize the effect of diffraction contact. A further reduction of the diffraction contrast can be obtained using a tilt series. A correlation between the intensity of the HASTEM signal and the real thickness value was found by comparing HASTEM and AFM images. HASTEM in combination with a tilt series can determine the material distribution in polycrystalline films and can accurately analyze 1-3 nm gaps of nanoelectrodes formed by electromigration.

\section{Introduction}

Characterization and understanding of failure mechanisms and electrical transport properties is crucial for the further development of micro- and nanoelectronics. Ten-nanometer-scale metallic wires are increasingly being used for such studies and applications. Passing an electric current through such wires can destroy them due to electromigration, which is a gradual displacement of atoms due to current passage [1].

Elsewhere we reported the possibility to follow the electromigration process in $\mathrm{Pt}$ [2] and in PtPd nanobridges [3] using in situ transmission electron microscopy (TEM) in bright-field TEM (BF-TEM) and scanning TEM (STEM) modes. In BF-TEM imaging of polycrystalline thin films, there are usually two competing types of contrast: mass-thickness contrast and diffraction contrast, which arises from the difference in orientation of the grains. This complicates the determination of the real thickness of such samples. Applying in situ STEM imaging of the electromigration process facilitates the study of mass transfer in polycrystalline films, but diffraction contrast can still influence the thickness analysis. 
In this paper we evaluate the use of the STEM method to analyze the thickness distribution in polycrystalline Pt-Pd nanobridges and show ways to minimize the diffraction contrast contribution to the STEM signal intensity.

\section{Material and methods}

Polycrystalline metal nanobridges with thicknesses of $15-20 \mathrm{~nm}$ were produced by sputtering Pd and Pt on 100-nm-thick freestanding silicon nitride membranes. Details of the sample fabrication process can be found in Refs. [2, 4, 5]. Each bridge is connected to 100-nm-thick gold contacts as shown in Figure 1a. We performed electromigration experiments in feedback-controlled (FBC) mode [6] and uniform voltage increase (UVI) mode prior to analyzing the thickness distribution in thin metal lines. The detailed description of in situ experiments and of the electrical measurements setup can be found in Refs. [2,7].

(S)TEM imaging was performed with a Titan FEI microscope operated at $300 \mathrm{kV}$ and equipped

with a Fischione 3000 annular dark-field detector (ADF). Samples were cooled to approximately $100 \mathrm{~K}$ to minimize carbon contamination. For atomic force microscopy (AFM) imaging we used a Veeco AFM Nanoscope V in tapping mode. 


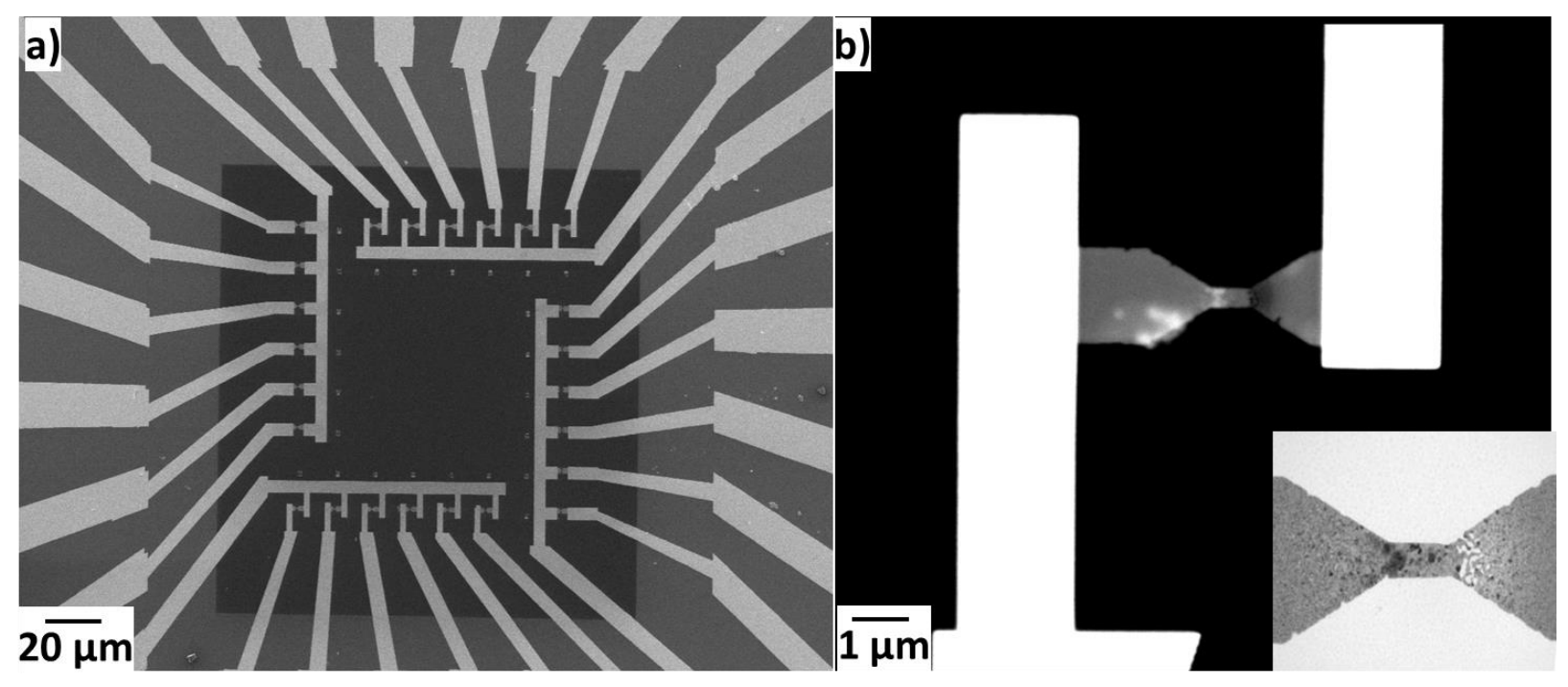

Figure 1. (a) SEM image of the central part of a $2 \times 2 \mathrm{~mm}^{2}$ chip with gold contacts and metal bridges located in the black area in the middle (freestanding membrane); (b) magnified SEM image of fully electromigrated and thus broken bridge; inset shows BF-TEM image of the same bridge.

\section{Results and discussion}

When an electric current is passed through the nanobridges, grain growth occurs prior to electromigration due to Joule heating. This effect can be seen in the $I-V$ plots as an upward change in the $I / V$ slope (see Figure 2a). Initially, all bridges that we investigated had a thickness of $15 \mathrm{~nm}$ and grain sizes of the as-fabricated bridges were 5-10 $\mathrm{nm}$. It is clear from Figure $1 \mathrm{~b}$ that bigger grains (20-50 $\mathrm{nm}$ in diameter) have formed in the center of the bridge and in adjacent contacts [8], whereas grains located far from the center remain unchanged. It was shown by Kozlova et al. [3] that electromigration in $\mathrm{Pt}-\mathrm{Pd}$ nanobridges displaces material from the cathode side towards the anode side of the bridge. Consequently this results in an inhomogeneity of the bridge thickness after the electromigration process has ended. 
Bridge breakage

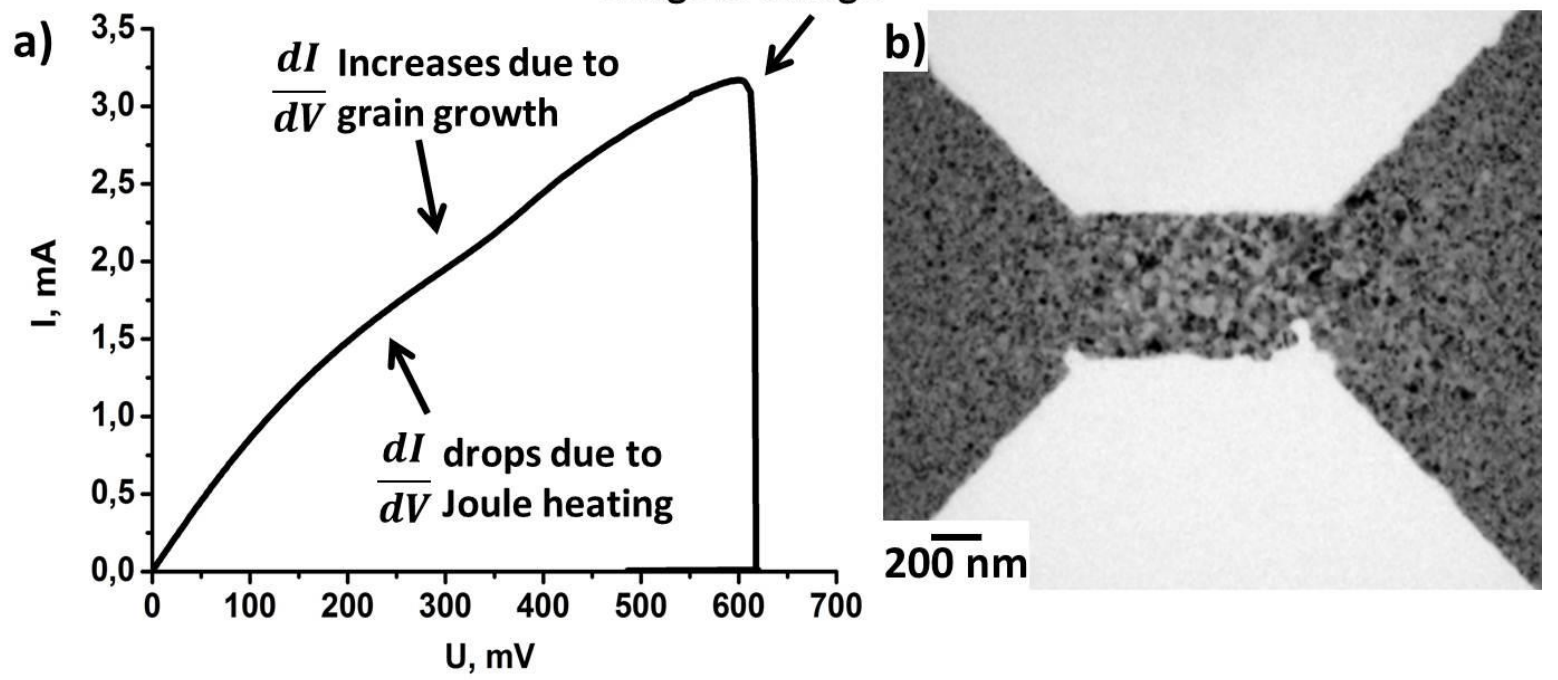

Figure 2. (a) Characteristic I-V profile of the in situ experiment. The initial increase in the differential resistance due to Joule heating is followed by a decrease induced by grain growth in the sample. Ongoing increase of the voltage leads to electromigration and subsequent bridge breakage. (b) Bright-field TEM image of the nanobridge after grain growth.

The analysis of mass transfer is hampered - as mentioned in the introduction - by diffraction contrast. Figure $2 \mathrm{~b}$ shows a bright-field image of a nanobridge after grain growth but before electromigration with still almost equal film thickness. The significant difference in contrast in this image is only due to diffraction contrast and thus it is clear that a bright-field image cannot be used to determine thickness. In order to minimize the effect of diffraction contrast, we obtained STEM images of the bridge after breakage with several values of camera lengths between 363 and $29 \mathrm{~mm}$. Images obtained at two outermost camera lengths are presented in Figures $3 \mathrm{a}$ (for $29 \mathrm{~mm}$ ) and Figure $3 \mathrm{~b}$ (for $363 \mathrm{~mm}$ ) at $0^{\circ} \alpha$ tilt angle. It is evident that, in the STEM image obtained at a camera length of $363 \mathrm{~mm}$ (Figure 3b), the gray levels not only reflect the thickness variation in the sample but also the grain orientation (low zone axis-oriented grains 
appear brighter). The STEM image acquired with a shorter camera length (Figure 3a) demonstrates much more uniform contrast on the sides of the sample where thickness is equal, although a small number of grains is still recognizable. Nevertheless the thickness variation in the sample can be determined from the variation in the STEM signal intensity (the brighter the area, the thicker it is).
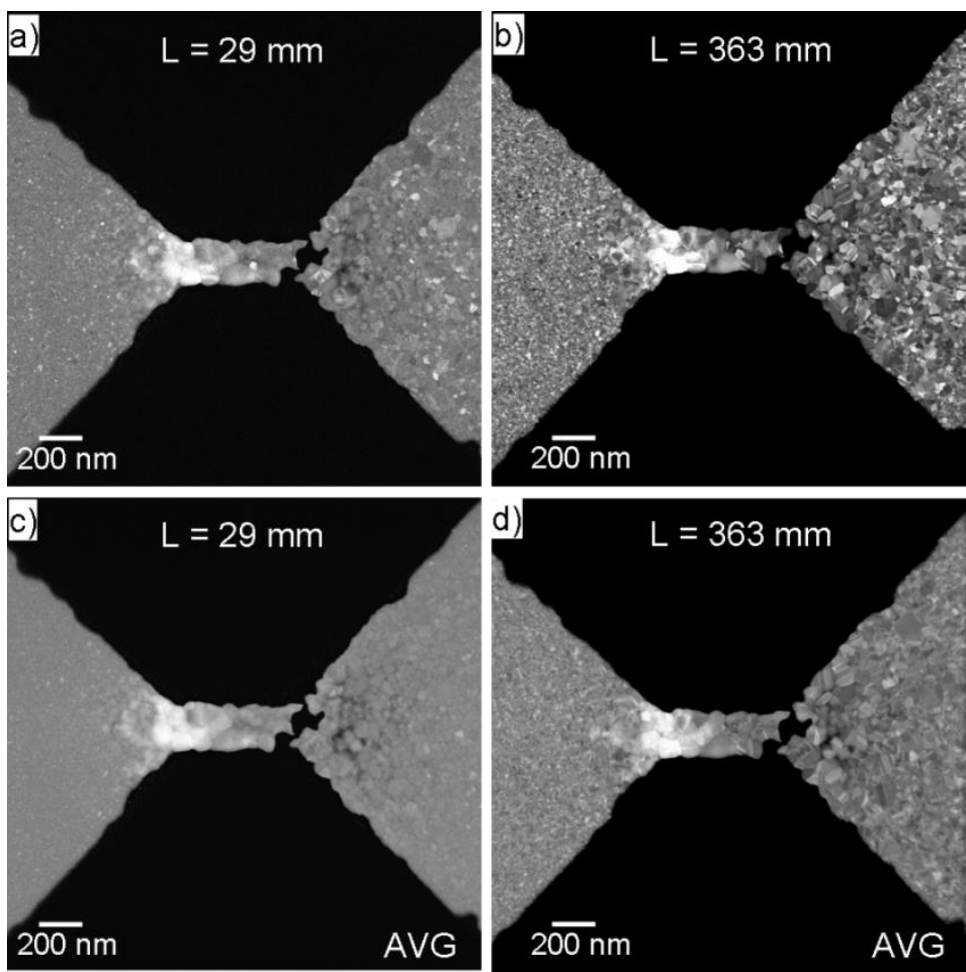

Figure 3. (a) - (b) STEM images of the bridge after breakage obtained for camera lengths of 29 and $363 \mathrm{~mm}$, respectively. Tilt angle is $0^{\circ} .(c)-(d)$ Averaged STEM images over the tilt series from $-5^{\circ}$ to $5^{\circ}$ with a step of $1^{\circ}$ for the same camera lengths.

The STEM images obtained for the smallest possible camera length of $29 \mathrm{~mm}$ still show some diffraction contrast, because a number of grains are visible. To further minimize the effect of the diffraction contrast in STEM, we recorded a tilt series for the same camera lengths with an $\alpha$ tilt 
from $-5^{\circ}$ to $5^{\circ}$ with steps of $1^{\circ}$. We aligned and averaged the obtained series of images using ImageJ $[9,10]$. The results of this averaging are presented in Figures $3 \mathrm{c}-\mathrm{d}$. For the short camera length $(29 \mathrm{~mm})$ the contrast in a contact area become almost uniform and individual grains are difficult to identify. For a camera length of $\mathrm{L}=363 \mathrm{~mm}$, the quality of a tilt-averaged image is similar to that of the single image obtained at $\mathrm{L}=29 \mathrm{~mm}$. It is evident that a tilt series is very useful for reducing the effect of diffraction contrast.

Whereas the use of the small camera length has a clear advantage of much less diffraction contrast, the STEM setting leads to much less counts on the HAADF detector and a longer exposure time is required to record each frame. For example for the in situ TEM recording of electromigration in nanobridges we used a frame rate of about $10-15$ frames/sec, whereas for quantitative analysis of the STEM frames, we used a rate of 3 frames/sec or less, thus slowing down the image frequency of the in situ study of the thickness variation.

The difference in the diffraction contrast for STEM images obtained at different camera lengths can in part be explained by considering the part of the diffraction pattern that is imaged on the HAADF detector. Figure 4 presents a line profile collected over the typical diffraction pattern for a polycrystalline Pt bridge (shown in the inset). The position of the inner radius of the ADF detector is superimposed over the line profile and indicated by red lines for camera lengths $L=$ $363 \mathrm{~mm}$ and $L=160 \mathrm{~mm}$. Note that, although there are no diffraction lines beyond $40 \mathrm{~nm}^{-1}$, diffraction contrast is still evident in the STEM images. This is due to the higher probability for incoherent scattering to large angles for the grains in a low-index orientation. Thus diffraction contrast cannot be prevented completely. 


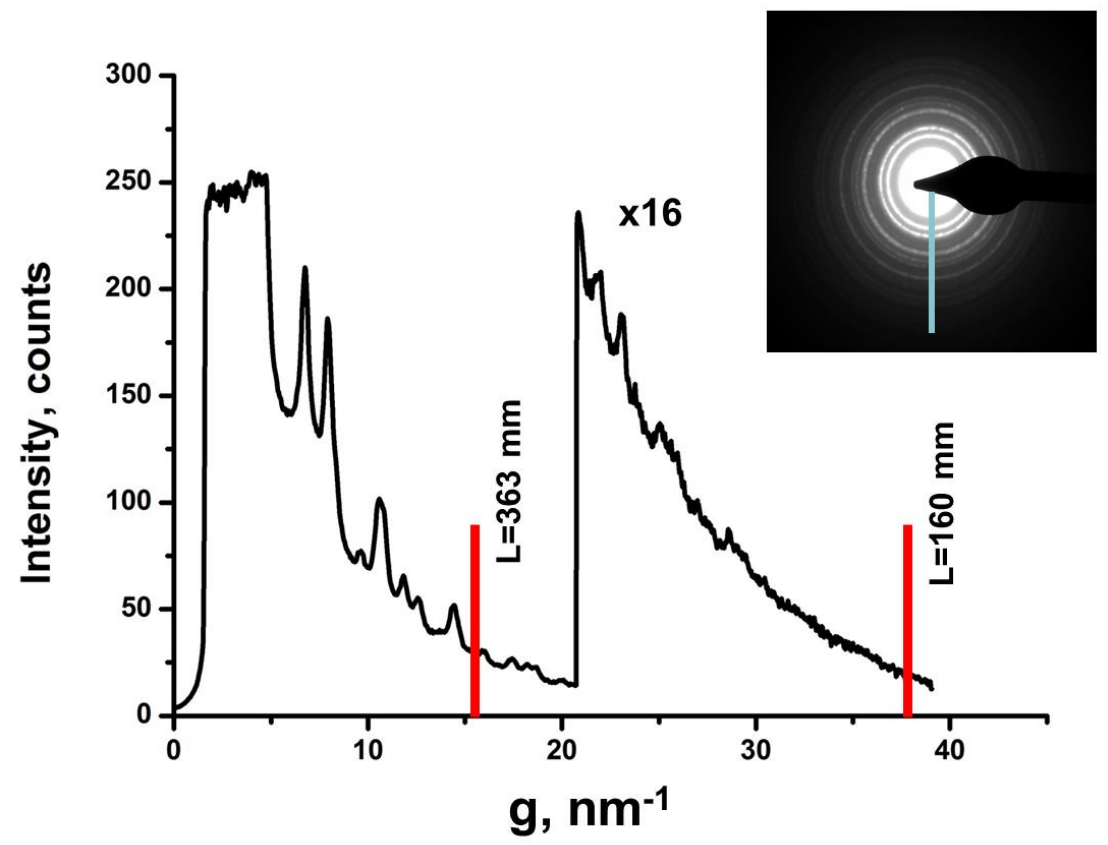

Figure 4. (a) Line profile collected over the diffraction pattern shown in the inset along red lines. Positions of the inner angle of the HAADF detector of 16 and 38 mrad correspond to a camera length of 363 and $160 \mathrm{~mm}$, respectively. The one obtained with a camera length of $29 \mathrm{~mm}(\sim 200$ mrad) used in our STEM images is not shown because it falls outside of this graph range.

In order to correlate directly the intensity in the STEM image and the local thickness of the metal film, we used an atomic force microscope (AFM) to image a nanobridge after electromigration (Figure 5a). The corresponding STEM image and 3D reconstruction of the bridge are presented in Figures $5 \mathrm{c}-\mathrm{d}$. As can be seen, the brightest area in the STEM image corresponds well to the area with the greatest thickness, whereas the area with voids corresponds to the region with the lowest intensity in the STEM image. Profiles obtained from an AFM image (Figure 5b) and the 3D reconstruction of the STEM image (Figure 5e) demonstrate a direct correlation between the height of the sample measured with an AFM tip and the intensity of the signal obtained with an HAADF detector. 


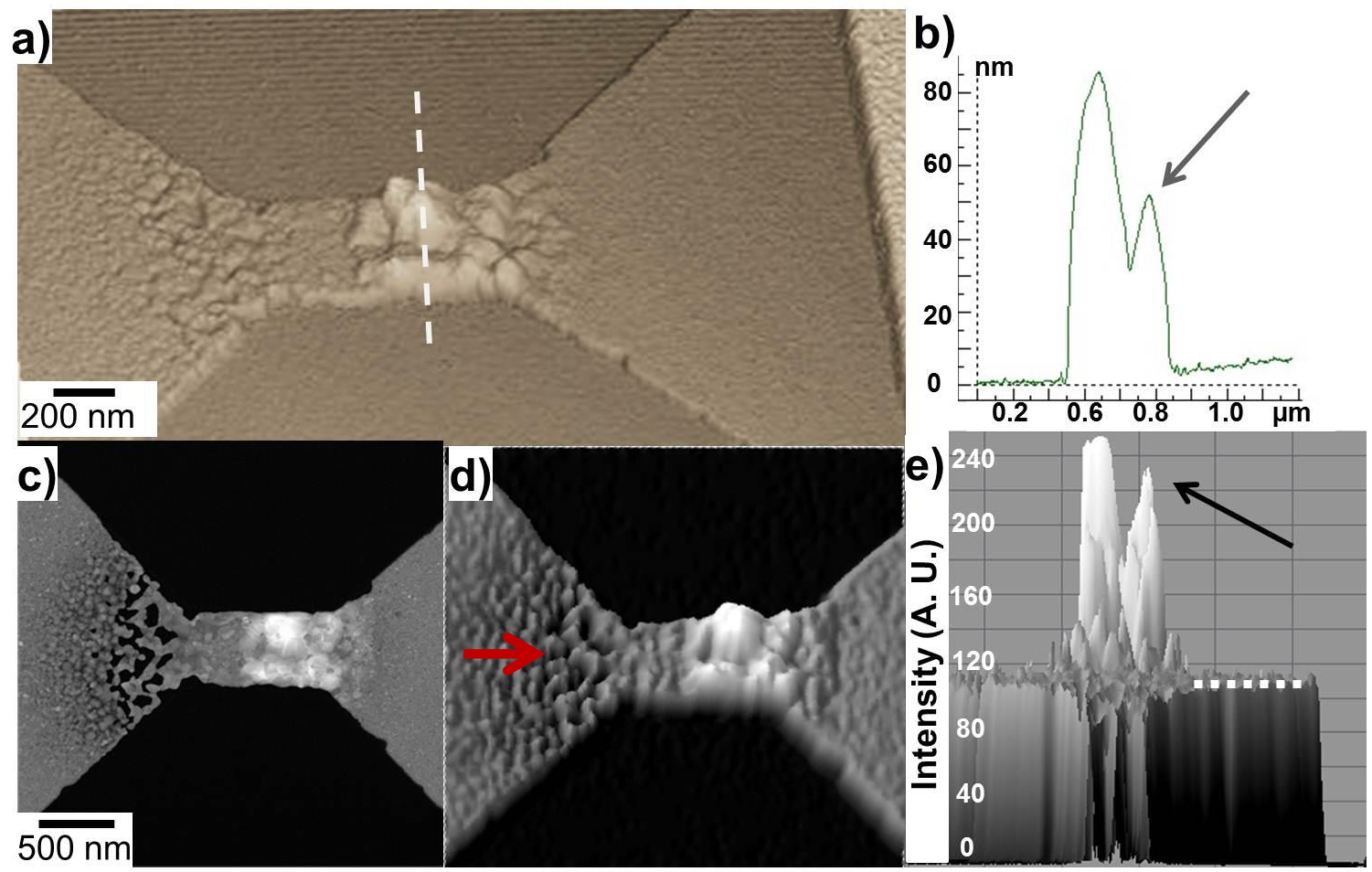

Figure 5. (a) 3D AFM image of the bridge showing the profile of material distribution after the electromigration process in the Pd-Pd bridge. (b) Thickness profile measured along the dashed line. (c) STEM image of the bridge; (d) 3D reconstruction of (c); (e) Side view of (d) from the direction indicated by the red arrow representing the height distribution. The dotted line corresponds to the original thickness of the sample, which is $20 \mathrm{~nm}$.

The direct correlation between the signal intensity in STEM imaging and the thickness of the sample can be used, for instance, to determine the material transfer due to electromigration [11] and for the detailed analysis of the shape of the formed nanogap. The analysis of the STEM image of a nanogap made in feedback control mode is shown in Figure 6. The height profile across the nanogap is shown in Figure $6 \mathrm{~b}$ whereas a 3D representation is shown in Figure 6c. It was demonstrated previously [5] that the use of FBC instead of UVI is more advantageous because it provides two symmetrical pointy contacts, which can be used, for example, for 
electrical transport measurements through single molecules [12]. In this type of study detailed knowledge of the exact shape of the nanogap is very useful. For instance, information about nanoelectrode separation as well the $3 \mathrm{D}$ profile of the gap allows the typical tunnel contact area of the junctions to be estimated. For the nanogap presented in Figure 6, the distance between electrodes is $2.4 \mathrm{~nm}$.
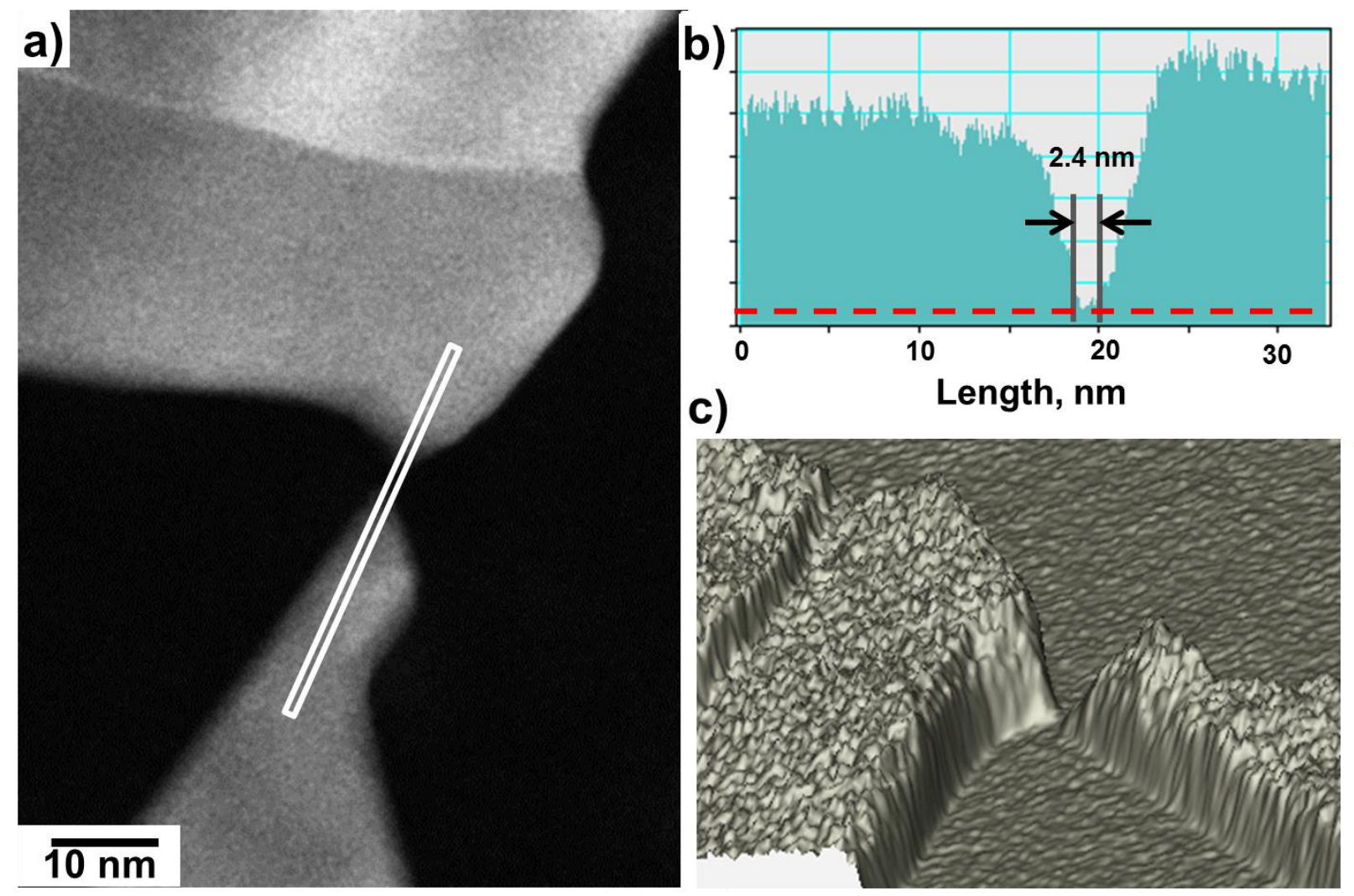

Figure 6. (a) STEM image of a nanogap obtained after the electromigration process. (b) Thickness profile reconstructed along the white line in (a) with a width of 10 pixels, dashed red line marks the background level. (c) 3D reconstruction of the nanogap.

The resolution of the AFM imaging of nanogaps is limited by the curvature of the scanning tip, which typically has a radius of $2 \mathrm{~nm}$ for ultrasharp tip [13]. Also the steepness of the 
nanoelectrodes complicates the accurate measurements of the nanogap depth. At the same time, scanning the sample with the focused electron beam in STEM mode allows more detailed information about the sample texture to be obtained.

\section{Conclusions}

A comparison of STEM images obtained at different camera lengths reveals that a careful choice of this parameter is vital for an accurate estimation of the sample thickness. In the case of relatively high camera lengths, the various grains in a polycrystalline samples are clearly visible, which complicates the analysis of the height profiles. When a sufficiently short (typically less than $40 \mathrm{~mm}$ ) camera length is used, the effect of the crystallographic orientation of the grains is small and this can be further minimized by averaging over a tilt series. In that case there is a good correlation between the intensity variation in the STEM image and the thickness distribution

obtained by AFM. Given the small widths of gaps in relation to the depth of the nanoelectrodes, STEM analysis provides a much more reliable thickness profile, for which an AFM tip is not sharp enough.

\section{Acknowledgments}

This research was supported by the Nano-Imaging under Industrial Conditions (NIMIC) project and the ERC grant.

\section{References}


1. Ho, P.S. and T. Kwok, Electromigration in Metals. Reports on Progress in Physics, 1989. 52(3): p. 301-348.

2. Gao, B., et al., In situ transmission electron microscopy imaging of grain growth in a platinum nanobridge induced by electric current annealing. Nanotechnology, 2011. 22(20).

3. Kozlova, T., Rudneva, M., Zandbergen, H. W, In-situ TEM and STEM studies of reversed electromigration in thin palladium-platinum bridges. Nano Letters, 2013.

4. Gao, B., et al., Three-terminal electric transport measurements on gold nano-particles combined with ex situ TEM inspection. Nanotechnology, 2009. 20(41).

5. Rudneva, M., In situ electrical measurements in transmission electron microscopy, 2013, Delft University of Technology.

6. Strachan, D.R., et al., Controlled fabrication of nanogaps in ambient environment for molecular electronics. Applied Physics Letters, 2005. 86(4).

7. Rudneva, M., et al., In-situ transmission electron microscopy imaging of electromigration in Pt nanowires. Microscopy and Microanalysis, 2012. 11.

8. McGarrity, K.S., B. Gao, and J.M. Thijssen, Simulation of Joule annealing in nanoscale Pt wires. Computational Materials Science, 2011. 50(10): p. 3043-3049.

9. Schneider, C.A., W.S. Rasband, and K.W. Eliceiri, NIH Image to ImageJ: 25 years of image analysis. Nature Methods, 2012. 9(7): p. 671-675.

10. ImageJ. Available from: http://imagejdocu.tudor.lu/.

11. Park, H., et al., Fabrication of metallic electrodes with nanometer separation by electromigration. Applied Physics Letters, 1999. 75(2): p. 301-303.

12. Prins, F., et al., Platinum-nanogaps for single-molecule electronics: room-temperature stability. Physical Chemistry Chemical Physics, 2011. 13(32): p. 14297-14301. 
13. Girod, S., et al., Real time atomic force microscopy imaging during nanogap formation by electromigration. Nanotechnology, 2012. 23(36). 